\title{
Synthesis of carbon-13 labelled oxalates exhibiting extended nuclear singlet state lifetimes
}

Lynda J. Brown ${ }^{\star}$, Giuseppe Pileio, Malcolm H. Levitt and Richard C. D. Brown

This is the peer reviewed version of the following article: "Synthesis of carbon-13 labelled oxalates exhibiting extended nuclear singlet state lifetimes", which has been published in final form at DOI 10.1002/jlcr.3479. This article may be used for non-commercial purposes in accordance with Wiley Terms and Conditions for Self-Archiving." 


\title{
Synthesis of carbon-13 labelled oxalates exhibiting extended nuclear singlet state lifetimes
}

\author{
Lynda J. Brown ${ }^{\star}$, Giuseppe Pileio, Malcolm H. Levitt and Richard C. D. Brown
}

Unsymmetrical perdeuterated doubly ${ }^{13} \mathrm{C}$ labelled oxalates exhibit extended lifetimes in the nuclear singlet state. Synthesis of $1,2{ }^{13} \mathrm{C}_{2}$ oxalates from commercially available precursors is described, facilitating preparation of unsymmetrical oxalates in a controlled manner.

Keywords: oxalates; hyperpolarized agents; carbon-13; deuterium; nuclear singlet state

\section{Introduction}

Magnetic resonance imaging (MRI) and nuclear magnetic resonance (NMR) detect signals that are inherently very weak; a consequence of the very small population differences between magnetically induced nuclear spin states. However, techniques such as dynamic nuclear hyperpolarisation (DNP) can deliver remarkable enhancement of NMR signal strength by modifying the distribution of nuclear spin states (or extent of polarisation) substantially away from equilibrium. The resulting increase in signal strength, which can exceed $10^{4}$ fold, opens up exciting opportunities for detection of trace intermediates in chemical and biochemical processes, such as metabolism. One factor that currently limits applications of DNP for spin one half nuclei (e.g. ${ }^{1} \mathrm{H}$ and ${ }^{13} \mathrm{C}$ ) is the rapid decay of hyperpolarisation over timescales of seconds, rather than the minutes that would be desirable. ${ }^{1-5}$ Recent efforts to extend the lifetime of hyperpolarisation include methods to create long-lived nuclear states that are protected from the efficient relaxation pathways present in the ground state. In suitably designed molecules containing a pair of coupled ${ }^{13} \mathrm{C}$ atoms, lifetime of the hyperpolarisation can be extended through conversion to a non-magnetic nuclear singlet state. ${ }^{6}$ The hyperpolarisation can then be read out by restoring the triplet state, offering the benefits of enhanced signal with lifetimes suitable for imaging applications. Thus, combining the DNP technique with doubly ${ }^{13} \mathrm{C}$ labelled organic molecules that support extended singlet lifetimes may offer exciting opportunities for in vivo NMR and MR imaging. However, a fundamental understanding of the structural features that support long-lived singlet states is key to future progress in this area and ultimately practical application. Here we describe the synthesis of unsymmetrically substituted oxalates containing two adjacent ${ }^{13} \mathrm{C}$ labels that have been shown to support long-lived nuclear singlet states.

A series of unsymmetrically substituted oxalates 4-6, that are doubly labelled with two adjacent ${ }^{13} \mathrm{C}$ atoms and contain per-deuterated side chains, have been synthesised and their use as long-lived repositories of enhanced NMR signal explored by preparing and observing the longlived nuclear spin order. ${ }^{7,8}$

*Department of Chemistry, University of Southampton, Highfield, Southampton, SO17 1BJ, UK, tel.: +44-(0)23-8059-7314; fax: +44-(0)23-8058-6805; e-mail: ljb2@ soton.ac.uk. 
The structure of the oxalate molecules had specific requirements (i) ${ }^{13} \mathrm{C}$ labelling of the two adjacent carbonyl carbons (ii) preferentially, all carbons neighbouring the carbonyls would possess deuterium only (iii) $R^{1}$ must be different to $R^{2}$ to break the symmetry of the molecule (figure 1). The importance of these design requirements has been discussed previously. ${ }^{7,9}$

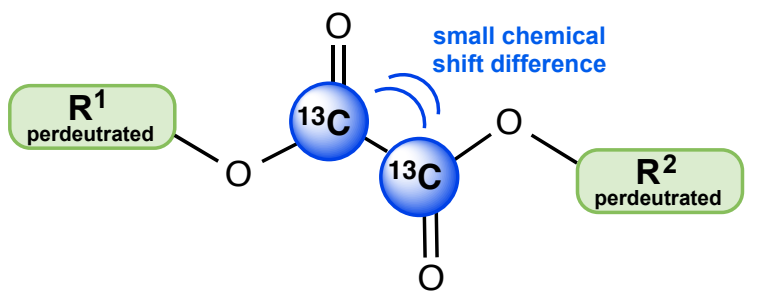

Figure 1: Structural requirements leading to extended nuclear singlet lifetimes $\left(T_{s}\right)$ for unsymmetrical oxalates

Herein we describe the preparation of oxalates that meet all the requirements described for extended singlet lifetimes.

\section{Experimental}

\section{General}

Oxalic acid- ${ }^{13} \mathrm{C}_{2}$ dihydrate and oxalyl chloride were purchased from Aldrich Chemical Co. and used without further purification. Deuterated alcohols were purchased from Cambridge Isotopes Ltd or QMX laboratories. All reactions were performed in an inert atmosphere in oven dried glassware. Chloroform and dichloromethane were dried by distillation from $\mathrm{CaCl}_{2}$ and $\mathrm{CaH}_{2}$ respectively. DMF (anhydrous) was purchased from Sigma Aldrich and used as supplied. ${ }^{1} \mathrm{H}$ $\mathrm{NMR}$ and ${ }^{13} \mathrm{C}$ NMR spectra were recorded in $\mathrm{CDCl}_{3}$ solution using Bruker AC300, AV300 (300 $\mathrm{MHz}$ and $75 \mathrm{MHz}$ respectively) or Bruker DPX $400(400 \mathrm{MHz}$ and $101 \mathrm{MHz}$ respectively) spectrometers. Chemical shifts are reported in $\delta$ units using chloroform as an internal standard ( $\delta=7.27 \mathrm{ppm}$ and $77.00 \mathrm{ppm}$ for ${ }^{1} \mathrm{H}$ and ${ }^{13} \mathrm{C}$, respectively). Infrared spectra were recorded on a Nicolet 380 spectrometer fitted with a Diamond platform, as solids or neat liquids. Melting points (mp) are uncorrected. Electron impact and chemical ionisation mass spectra were obtained using a Fisons VG platform single quadropole mass spectrometer. Electrospray mass spectra were obtained using a Micromass platform mass analyser with an electrospray ion source.

Cyclohexyl- $d_{11}$ (propan-2-yl- $d_{7}$ ) oxalate- ${ }^{13} \mathrm{C}_{2}$ (4) (General Procedure for synthesis of oxalates 4 $-6)$

To oxalic acid- ${ }^{13} \mathrm{C}_{2}$ dihydrate $(250 \mathrm{mg}, 1.95 \mathrm{mmol})$ suspended in anhydrous $\mathrm{CHCl}_{3}(1 \mathrm{~mL})$ was added isopropanol- $d_{8}\left(597 \mu \mathrm{L}, 7.80 \mathrm{mmol}, 4.0\right.$ equiv.) and conc. $\mathrm{H}_{2} \mathrm{SO}_{4}(208 \mu \mathrm{L}, 3.90 \mathrm{mmol}, 2.0$ equiv.) and the reaction heated under gentle reflux for $2 \mathrm{~h}$. The reaction mixture was purified directly by silica gel column chromatography eluting with $\mathrm{Et}_{2} \mathrm{O}$ :pentane (1:5) to give diisopropyl$d_{7}$ oxalate- ${ }^{13} \mathrm{C}_{2}(7)$ as a colourless oil (348 $\mathrm{mg}, 1.83 \mathrm{mmol}, 94 \%$ ).

To diisopropyl- $d_{7}$ oxalate- ${ }^{13} \mathrm{C}_{2}$ (330 mg, $1.74 \mathrm{mmol}$ ) and $\mathrm{KHCO}_{3}(174 \mathrm{mg}, 1.74 \mathrm{mmol}, 1.0$ equiv.) was added $\mathrm{H}_{2} \mathrm{O}(2 \mathrm{~mL})$ and the reaction heated at $60{ }^{\circ} \mathrm{C}$ for $2 \mathrm{~h}$. The reaction was allowed to cool to $\mathrm{rt}$ and acetone $(30 \mathrm{~mL})$ added. The mixture was cooled on ice for 10 min during which 
time a white precipitate formed which was removed by filtration, washed with acetone $(3 \times 10$ $\mathrm{mL}$ ) and dried in vacuo to give potassium 2-isopropoxy- $d_{7}-2$-oxoacetate- $-{ }^{13} \mathrm{C}_{2}(8)$ as a white solid (211 mg, $1.18 \mathrm{mmol}, 68 \%$ ); $\mathrm{mp} 215-220^{\circ} \mathrm{C}$.

To potassium 2-isopropoxy- $d_{7}$-2-oxoacetate- ${ }^{13} \mathrm{C}_{2}(205 \mathrm{mg}, 1.14 \mathrm{mmol})$ suspended in anhydrous $\mathrm{CH}_{2} \mathrm{Cl}_{2}(2 \mathrm{~mL})$ at $\mathrm{rt}$, under an atmosphere of nitrogen, was added oxalyl chloride $(97 \mu \mathrm{L}, 1.14$ mmol, 1.0 equiv.) dropwise to control effervescence. After $5 \mathrm{~min}$, DMF $(8.5 \mu \mathrm{L}, 0.11 \mathrm{mmol}, 0.1$ equiv.) was added causing further effervescence. The reaction was stirred for $1 \mathrm{~h}$ and then cyclohexanol- $d_{12}$ was added (131 $\mu \mathrm{L}, 1.25 \mathrm{mmol}, 1.1$ equiv.) and the reaction stirred for a further $20 \mathrm{~min}$. The reaction was purified directly by silica gel column chromatography eluting with $\mathrm{Et}_{2} \mathrm{O}$ :pentane (1:4) to give cyclohexyl- $d_{11}$ (propan-2-yl- $d_{7}$ ) oxalate- ${ }^{13} \mathrm{C}_{2}(4)$ as a colourless oil (160 mg, $0.68 \mathrm{mmol}, 60 \%$; $35 \%$ over three steps).

Cyclohexyl isopropyl oxalate:

$v_{\max }$ (neat) $\mathrm{cm}^{-1} ; 2983,1735,1305,1178 ; \delta_{\mathrm{H}}\left(300 \mathrm{MHz} ; \mathrm{CDCl}_{3}\right) 5.13$ (sept, $J=6.2 \mathrm{~Hz}, 1 \mathrm{H}$, $\left.\left(\mathrm{CH}_{3}\right)_{2} \mathrm{CH}\right), 4.89\left(\mathrm{~m}, 1 \mathrm{H}, \mathrm{CHCH}_{2}\right), 1.90\left(\mathrm{~m}, 2 \mathrm{H}, \mathrm{CH}_{2}\right), 1.82-1.69\left(\mathrm{~m}, 2 \mathrm{H}, \mathrm{CH}_{2}\right), 1.58-1.23(\mathrm{~m}$, $\left.6 \mathrm{H}, \mathrm{CH}_{2}\right), 1.34\left(\mathrm{~d}, J=6.2 \mathrm{~Hz}, 6 \mathrm{H},\left(\mathrm{CH}_{3}\right)_{2} \mathrm{CH}\right) ; \delta_{\mathrm{C}}\left(75 \mathrm{MHz} ; \mathrm{CDCl}_{3}\right)$ 157.82, 157.71, 75.95, 71.15, 31.15, 25.10, 23.59, 21.45; GC-MS (Cl) m/z $232\left(\left[\mathrm{M}+\mathrm{NH}_{4}\right]^{+}\right)$.

Cyclohexyl- $d_{11}$ (propan-2-yl- $\left.d_{7}\right)$ oxalate- ${ }^{13} \mathrm{C}_{2}(4)$ :

$\delta_{\mathrm{C}}\left(75 \mathrm{MHz} ; \mathrm{CDCl}_{3}\right)$ 157.88; GC-MS (Cl) m/z $252\left(\left[\mathrm{M}+\mathrm{NH}_{4}\right]^{+}\right)$.

Ethyl methyl oxalate:

$v_{\max }$ (neat) $\mathrm{cm}^{-1} ; 2987,1739,1317,1151 ; \delta_{\mathrm{H}}\left(300 \mathrm{MHz} ; \mathrm{CDCl}_{3}\right) 4.35$ (q, J=7.2 Hz, 2H, $\left.\mathrm{CH}_{2}\right)$, $3.89\left(\mathrm{~s}, 3 \mathrm{H}, \mathrm{OCH}_{3}\right), 1.37$ (t, $\left.J=7.2 \mathrm{~Hz}, 3 \mathrm{H}, \mathrm{CH}_{3}\right) ; \delta_{\mathrm{C}}\left(75 \mathrm{MHz} ; \mathrm{CDCl}_{3}\right)$ 158.19, 157.50, 63.16, 53.44, 13.84; GC-MS (Cl) m/z $150\left(\left[\mathrm{M}+\mathrm{NH}_{4}\right]^{+}\right)$.

Ethyl- $d_{5}$ (methyl- $d_{3}$ ) oxalate- ${ }^{13} \mathrm{C}_{2}(\mathbf{5})$ :

$\delta_{\mathrm{C}}\left(101 \mathrm{MHz} ; \mathrm{CDCl}_{3}\right) 159.03,158.03,157.81,156.81\left(\mathrm{ABq}, 2 \mathrm{C}, \Delta \delta_{\mathrm{AB}}=68.9 \mathrm{~Hz}, J_{\mathrm{AB}}=101.0 \mathrm{~Hz}\right)$ GC-MS (EI) m/z $63\left(\left[\mathrm{C}^{13} \mathrm{O}_{2} \mathrm{CD}_{3}\right]^{+}, 100 \%\right), 50\left(\left[\mathrm{C}_{2} \mathrm{D}_{5} \mathrm{O}\right]^{+}, 27 \%\right)$.

Isopropyl methyl oxalate:

$v_{\max }$ (neat) $\mathrm{cm}^{-1} ; 2988,1738,1317,1150 ; \delta_{\mathrm{H}}\left(400 \mathrm{MHz} ; \mathrm{CDCl}_{3}\right) 5.15$ (sept, $J=6.6 \mathrm{~Hz}, 1 \mathrm{H}$, $\left.\left(\mathrm{CH}_{3}\right)_{2} \mathrm{CH}\right), 3.87\left(\mathrm{~s}, 3 \mathrm{H}, \mathrm{CH}_{3}\right), 1.34\left(\mathrm{~d}, J=6.6 \mathrm{~Hz}, 6 \mathrm{H},\left(\mathrm{CH}_{3}\right)_{2} \mathrm{CH}\right) ; \delta_{\mathrm{C}}\left(101 \mathrm{MHz} ; \mathrm{CDCl}_{3}\right)$ 158.45, 157.11, 71.50, 53.33, 21.41; GC-MS (EI) m/z $59\left(\left[\mathrm{C}_{3} \mathrm{H}_{7} \mathrm{O}\right]^{+}, 64 \%\right), 43\left(\left[\mathrm{C}_{3} \mathrm{H}_{7}\right]^{+}, 100 \%\right)$.

Methyl- $d_{3}$ (propan-2-yl- $d_{7}$ ) oxalate- ${ }^{13} \mathrm{C}_{2}(6)$ :

$\delta_{\mathrm{C}}\left(101 \mathrm{MHz} ; \mathrm{CDCl}_{3}\right) 159.20,158.20,157.53,156.53\left(\mathrm{ABq}, 2 \mathrm{C}, \Delta \delta_{\mathrm{AB}}=133.7 \mathrm{~Hz}, J_{\mathrm{AB}}=101.0\right.$ $\mathrm{Hz}) ; \mathrm{GC}-\mathrm{MS}(\mathrm{Cl}) \mathrm{m} / \mathrm{z} 176\left(\left[\mathrm{M}+\mathrm{NH}_{4}\right]^{+}\right)$.

\section{Results and discussion}

${ }^{13} \mathrm{C}_{2}$-Oxalyl chloride was not considered to be a suitable starting material for the synthesis of unsymmetrically substituted oxalates containing perdeuterated alkyl groups due to practical challenges related to its stability, and in terms of isolation and characterisation of sensitive intermediates resulting low yields and poor reproducibility. Additionally, oxalyl chloride whilst convenient for the synthesis of symmetrical diesters, gives rise to mixtures and challenging separations if directly applied to the synthesis of unsymmetrical oxalates. An alternative approach involved refluxing oxalic acid with, equimolar quantities of two alcohols under acidic $\left(\mathrm{H}_{2} \mathrm{SO}_{4}\right)$ conditions, furnishing mixtures of oxalates. ${ }^{10}$ However isolation of pure products 
demanded time-consuming chromatography especially where there was very little structural difference between the ester groups. A different strategy involving preparation of ${ }^{13} \mathrm{C}$ labelled oxalates followed by perdeutration of the side chains by hydrogen-deuterium exchange was considered. ${ }^{11}$ Hydrogen-deuterium exchange can be carried out on advanced intermediates, and in principle, save time and cost. However, this method can be hampered by problems of non-specific labelling which leads to mixtures of isotopomers and isotopologues, and as the main cost is the ${ }^{13} \mathrm{C}$ labelled material, this approach was not pursued.

It was concluded that an improved regioselective synthesis of this class of compounds was required. Burrell et al. reported a synthesis of ${ }^{14} \mathrm{C}_{2}$ labelled methyl 2-chloro-2-oxoacetate as a convenient labelled oxalyl chloride equivalent by means of selective partial hydrolysis of labelled dimethyl oxalate, and showed its synthetic utility in the synthesis of ${ }^{14} \mathrm{C}_{2}$ labelled dichloropyrazinones. ${ }^{12}$ Elaboration of this chemistry provided a simple, high yielding route to unsymmetrical ${ }^{13} \mathrm{C}$ deuterated or perdeuterated oxalates. This method not only prevents the formation of mixtures but also allows the stepwise introduction of ${ }^{13} \mathrm{C}$ or deuterium labels at different positions within the molecule.

Our synthetic approach utilised commercially available oxalic acid- ${ }^{13} \mathrm{C}_{2}$ dihydrate (1), which was heated under reflux with excess alcohol (deuterated, partially deuterated or unlabelled) in chloroform with $\mathrm{H}_{2} \mathrm{SO}_{4}$ to provide the symmetrical oxalates. The symmetrical oxalates underwent selective mono-hydrolysis using one equivalent of $\mathrm{KHCO}_{3}$ to give the potassium salts 2 which were isolated in most cases as white precipitates. Reaction of potassium salts 2 with one equivalent ${ }^{\mathrm{a}}$ of oxalyl chloride provided the acid chlorides which were reacted directly (one-pot) with a small excess of the required second alcohol. Under these optimised conditions the unsymmetrical oxalates 3 were obtained in high yields (scheme 1).
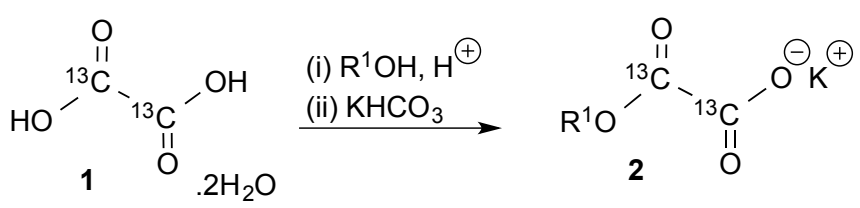

(i) $(\mathrm{COCl})_{2}$

$\stackrel{\text { (ii) } \mathrm{R}^{2} \mathrm{OH}}{\longrightarrow}$

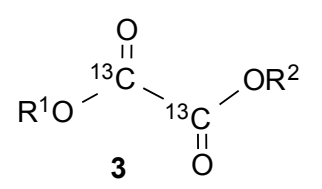

$4 \mathrm{R}^{1}=\left(\mathrm{CD}_{3}\right)_{2} \mathrm{CD}$ $\mathrm{R}^{2}=\mathrm{C}_{6} \mathrm{D}_{11}$

$\begin{aligned} \mathrm{R}^{1} & =\mathrm{CD}_{3} \\ \mathrm{R}^{2} & =\mathrm{C}_{2} \mathrm{D}_{5}\end{aligned}$

$\mathrm{R}^{1}=\mathrm{CD}_{3}$

$6 \mathrm{R}^{2}=\left(\mathrm{CD}_{3}\right)_{2} \mathrm{CD}$

Scheme 1: General synthesis of unsymmetrical oxalates 4-6

Scheme 2 describes this synthesis applied to the preparation of cyclohexyl- $d_{11}$ (propan-2-yl- $d_{7}$ ) oxalate $-{ }^{13} \mathrm{C}_{2}$ (4) in $38 \%$ overall yield for the three steps. Following this a further three unsymmetrical unlabelled oxalates were prepared permitting full characterisation, and based on these results their fully labelled analogues were also synthesised.

\footnotetext{
${ }^{a}$ It is important to avoid the use of excess of oxalyl chloride, which if present in the crude product, would give the symmetrical oxalate $\left(\mathrm{R}^{2} \mathrm{O}(\mathrm{CO})_{2} \mathrm{OR}{ }^{2}\right)$, leading to a challenging purification.
} 


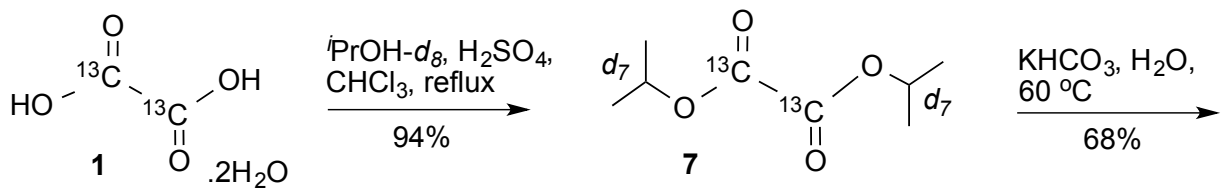

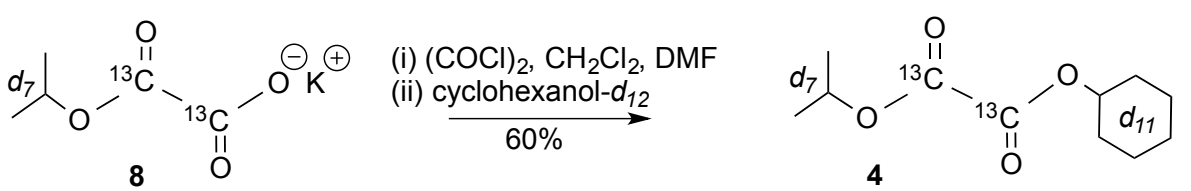

Scheme 2: Synthesis of cyclohexyl- $d_{11}$ (propan-2-yl- $d_{7}$ ) oxalate- ${ }^{13} C_{2}$ (4)

In the case of the doubly ${ }^{13} \mathrm{C}$ labelled mixed oxalate diesters $4-6$, the ${ }^{13} \mathrm{C}$ NMR spectra for the two adjacent ${ }^{13} \mathrm{C}$ nuclei show second order effects due to the similarities in the chemical shift differences and the coupling constants $J_{1 c-c}$. This is most notable for the natural abundance and ${ }^{13} \mathrm{C}_{2}$ labelled cyclohexyl isopropyl oxalates (figure 2), where natural abundance cyclohexyl isopropyl oxalate clearly presents two resonances with a chemical shift difference of $0.11 \mathrm{ppm}$ (top spectrum), whereas the ${ }^{13} \mathrm{C}$ NMR spectrum of cyclohexyl- $d_{11}$ (propan-2-yl- $d_{7}$ ) oxalate- ${ }^{13} C_{2}$ shows only a single peak (bottom spectrum). In the case of the ethyl methyl oxalate the chemical shift difference of the two adjacent ${ }^{13} \mathrm{C}$ nuclei is greater $(0.69 \mathrm{ppm})$ and the spectrum of ethyl- $d_{5}$ (methyl- $d_{3}$ ) oxalate- ${ }^{13} \mathrm{C}_{2}$ presents an $\mathrm{AB}$ system as the chemical shift difference is still less than that of the $J$ coupling $\left(\Delta \delta_{A B}=68.9 \mathrm{~Hz}, J_{A B}=101.0 \mathrm{~Hz}\right)$.

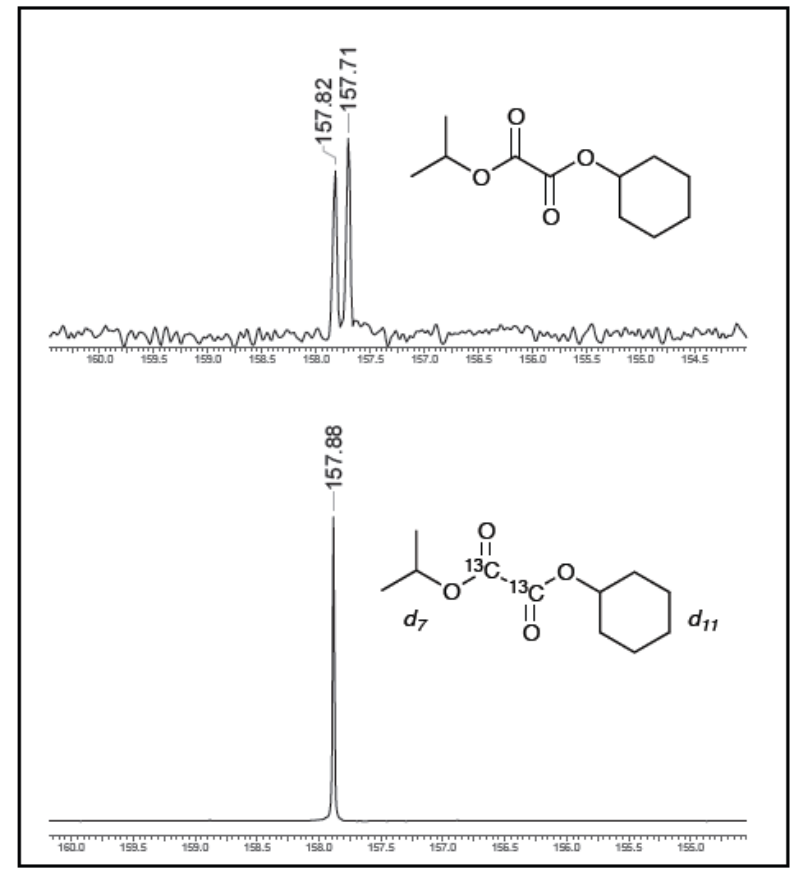

(i)

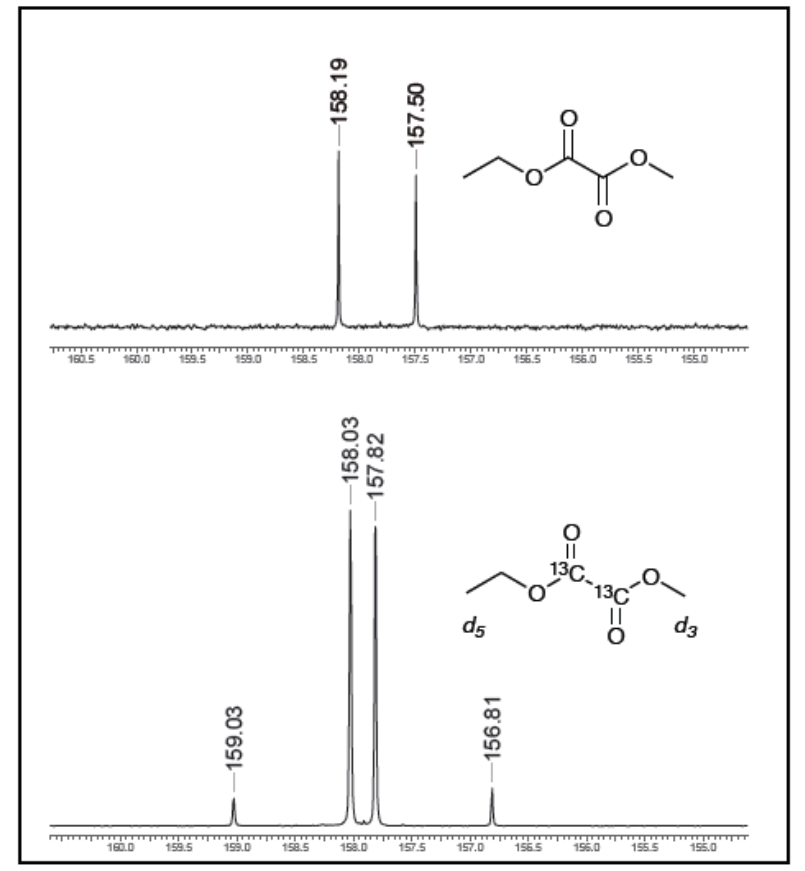

(ii) 
Figure 2: ${ }^{13} \mathrm{C}$ NMR spectra of (i) natural abundance cyclohexyl isopropyl oxalate (top) and labelled cyclohexyl- $d_{11}$ (propan-2-yl- $d_{7}$ ) oxalate- ${ }^{13} C_{2}(4)$ (bottom) and (ii) natural abundance ethyl methyl oxalate (top) and labelled ethyl- $d_{5}$ (methyl- $d_{3}$ ) oxalate $-{ }^{13} C_{2}(5)$ (bottom).

In summary, a practical synthesis of unsymmetrical oxalate diesters has been described, which allows for incorporation of adjacent ${ }^{13} \mathrm{C}$ labels and perdeuterated ester side chains with economic use of the expensive ${ }^{13} \mathrm{C}$ labelled starting materials. The labelled unsymmetrically substituted oxalate derivatives cyclohexyl- $d_{11}$ (propan-2-yl- $d_{7}$ ) oxalate- ${ }^{13} C_{2}(4)$ and methyl- $d_{3}$ (propan-2-yl- $d_{7}$ ) oxalate $-{ }^{13} \mathrm{C}_{2}$ (6) have exhibited singlet lifetimes of 244 seconds and 154 seconds respectively helping to advance progress and understanding needed to develop longlived states for applications in hyperpolarised NMR. According to our design principles, these compounds represent our first generation of molecules containing adjacent ${ }^{13} \mathrm{C}$ pairs that exhibit long-lived nuclear spin order. This has led to the development of subsequent generations of acetylenes ${ }^{7,13}$ and napthalenes with singlet lifetimes of greater than 1 hour in room temperature solution., ${ }^{9,14}$

\section{Acknowledgements}

The authors thank The Royal Society for the award of a Dorothy Hodgkin Research Fellowship (LJB) and acknowledge financial support from European Regional Development Fund (ERDF), ISCE-Chem INTERREG IV A France-(Channel)-England project no. 4061, the EPSRC-UK (EP/N002482 and EP/K039466/1), the European Research Council (291044 - HYPERSINGLET) and the Wolfson Foundation (WM110069).

\section{References}

[1] J. H. Ardenkjaer-Larsen, B. Fridlund, A. Gram, G. Hansson, L. Hansson, M. H. Lerche, R. Servin, M. Thaning, K. Golman, Proc. Natl. Acad. Sci. USA 2003, 100, 10158-10158.

[2] G. Pileio, M. Carravetta, M. H. Levitt, Proc. Natl. Acad. Sci. USA 2010, 107, 1713517139.

[3] M. Carravetta, O. G. Johannessen, M. H. Levitt, Phys., Rev. Lett. 2004, 92, 153003.

[4] M. Carravetta, M. H. Levitt, J. Chem. Phys. 2005, 122, $214505-214514$.

[5] R. K. Ghosh, S. J. Kadlecek, J. H. Ardenkjaer-Larsen, B. M. Pullinger, G. Pileio, M. H. Levitt, N. N. Kuzma, R. R. Rizi, Magn. Reson. Med. 2011, 66, 1177-1180.

[6] M. H. Levitt, Annu. Rev. Phys. Chem. 2012, 63, 89-105.

[7] C. Laustsen, G. Pileio, M. C. D. Tayler, L. J. Brown, R. C. D. Brown, M. H. Levitt, J. H. Ardenkjaer-Larsen, Magn. Reson. Med. 2012, 68, 1262-1265.

[8] G. Pileio, J. T Hill-Cousins, S. Mitchell, I. Kuprov, L. J. Brown, R. C. D. Brown, M. H. Levitt, J. Am. Chem. Soc. 2012, 134, 17494-17497.

[9] J.T. Hill-Cousins, I.-A. Pop, G. Pileio, G. Stevanato, P. Hakansson, S.S. Roy, M.H. Levitt, L.J. Brown, R.C.D. Brown, Org. Lett. 2015, 17, 2150-2153.

[10] E. Bowden, Org Synth. Coll. Vol 2, 1943, 414.

[11] V. Derdau, J. Atzrodt, J. Zimmermann, C. Kroll, F. Brueckner, Chem. Eur. J. 2009, 15, 10397-10404.

[12] R. C. Burrell, J. A. Easter, S. J. Bonacorsi, Jr., B. Balasubramanian, J. Labelled Comp. Radiopharm. 2009, 52, 549-552. 
[13] G. Pileio, S. Bowen, C. Laustsen, M. C. D. Tayler, J. T. Hill-Cousins, L. J. Brown, R. C. D. Brown, J. H. Ardenkjaer-Larsen, M. H. Levitt, J. Am. Chem. Soc., 2013, 135, 5084-5088.

[14] G. Stevanato, J. T. Hill-Cousins, P. Håkansson, S. S. Roy, L. J. Brown, R. C. D. Brown, G. Pileio, M. H. Levitt, Solution, Angew. Chem. Int. Ed. 2015, 54, 3740-3743. 